\title{
Mixed Perspectives and Thematic Analysis in Design Education
}

\author{
YU Qinghua ${ }^{\dagger}$ and Yukari Nagai ${ }^{*}$
}

\section{Abstract}

This research explores the design of products based on users' emotional requirements and how students can be stimulated to generate novel ideas in design education. In order to achieve these aims, multiple methods were taught to students during an online course. In the first step, the students utilised interviews, questionnaires, and mixed perspectives to design hill censers according to the users' emotional requirements. In the second step, the researcher conducted a qualitative thematic analysis to study the students' collected survey reports. The analytic results were then shared with students to help them quickly obtain better novel design ideas. And then, an emotional design appraisal model was built in the third step. The two main findings are as follows: first, creation in light of the stakeholder's perspective enabled the students to come up with better design ideas quickly. Second, the 'design method' and 'emotional experience' themes obtained by the thematic analysis were found to be vital for the designers/students. Notably, the 'design method' theme can help students generate novel design ideas, and the students can learn the users' needs from the 'emotional experience' theme.

Keywords: Mixed Perspectives; Thematic Analysis; Emotional Requirements; Hill Censer; Design Education; Japan

\footnotetext{
† Doctoral Student, Japan Advanced Institute of Science and Technology, Knowledge Science School, Japan Advanced Institute of Science and Technology, 1-1 Asahidai, Nomi, Ishikawa, 923-1292, Japan \& An Associate Professor, Jingdezhen Ceramic Institute, China, Email: yuqinghua@jaist.ac.jp

¥ Professor, Japan Advance Institute of Science and Technology, Knowledge Science School, Japan Advanced Institute of Science and Technology, 1-1 Asahidai, Nomi, Ishikawa 923-1292, Japan,

${ }^{*}$ Corresponding Author, E-mail: ynagai@jaist.ac.jp

(C)2021 Qinghua \& Nagai. This is an Open Access article distributed under the terms of the Creative Commons Attribution License (http://creativecommons.org/licenses/by/2.0), which permits unrestricted use, distribution, and reproduction in any medium, provided the original work is properly cited.
} 


\section{Introduction}

This research employed multiple methods in studying design through the case study of designing products (hill censers). The case study was conducted with students taking an online product design course at the Jingdezhen Ceramic Institute, Jiangxi province, China. Specifically, this study aimed at helping students design products based on users' emotional requirements stimulating them to easily generate novel ideas. It has been shown that emotions can deeply influence the overall user experience (Van Gorp \& Adams, 2012), so understanding the users' feelings and emotions is vital in the design process (Sáenz et al., 2019). In addition, the generation of novel ideas is a vital and challenging part of the creative design process (Shroyer et al., 2018).

This online course taught the students to utilise multiple methods to learn the users' emotional requirements and the built emotional design appraisal model proposed here can also be used in future design courses to guide students in quickly coming up with innovative design ideas based on the users' emotional requirements. In order to achieve this goal, this research focused on answering two research questions. The first question asked how products could be designed according to users' emotional requirements, and the second asked how designers could be assisted in quickly coming up with design ideas.

Knowing enough about the history and background of the product being designed is an essential first step in the design process. In this research, the hill censer, one of the typical historical items in Chinese incense burning, was chosen as the product. A hill-shaped censer is originated under the reign of Emperor Wudi (140-87 BC) of the Han Dynasty in China. It has a special status in the history of the censers. However, it has not been prevalent since the Wei-jin period (220-420 AD). As hill censers have evolved in form and remain prevalent nowadays, they deserve further study. The name of the hill censer is based on the fairy sea hill form it takes. The hill-shaped lids initially showed images of hunting and hiding in the hills (Lian, 2013). The most representative forms show auspicious beasts trying to hide while hunters with bows and arrows attempt to find them. Hill-shaped censers originated during the Emperor Wudi period (156-87 BC) of the Han Dynasty (206 BC220 AD). This kind of hill-shaped censer has been prevalent ever since that time.

The remainder of this paper is organised as follows. The next section presents the leading design and research methods used in this study, while the section following the section on research design presents the details of the hill censer's design course for the case study. The succeeding section presents the findings obtained by employing mixed perspectives and the thematic analysis method. The designs of the case study are evaluated following this. While the penultimate section an emotional design appraisal model, the final section concludes narrating the two main findings.

\section{Research Methods}

All classes were held online to replace the initially planned regular courses due to the COVID-19 pandemic. The process of the online course was divided into three steps, as shown below.

Mixed perspectives and thematic analysis were the main approaches utilised in this case. The former was used by the students in their design process, as it is the most vital method to learn about the users' emotional requirements. The latter was leveraged by the researcher to analyse the survey reports of the students, after which the analytical results were shared with the students to help them generate innovative design ideas. In addition to these two methods, the students conducted interviews and administered questionnaires to collect data on users' emotional requirements.

\section{Process of the Online Course}

The schematic process followed in this study is shown in Figure 1.

- In the first step, the researcher prepared the material for live online lectures, which the students were required to 
attend. During these lectures, the students learned multiple methods in product design.

- The second step consisted of analysis and evaluation shown in the yellow rectangle, which started with giving assignments to the students. For the first assignment, the students had to interview the censer's users and write a survey report after interviewing. The researcher analysed the collected survey reports and provided feedback to the students about the nine common themes summarised from all the survey reports. The second assignment required that the students design a product (hill censer) based on the survey report they had prepared using the techniques they learned in the online lectures. Design professors and users of the product (censer) evaluated the final designs of the students.

- In the third step, design professors (including the researcher) built an emotional design appraisal model, which will be utilised in the students' future designs to help them to quickly generate innovative design ideas based on the users' emotional requirements.

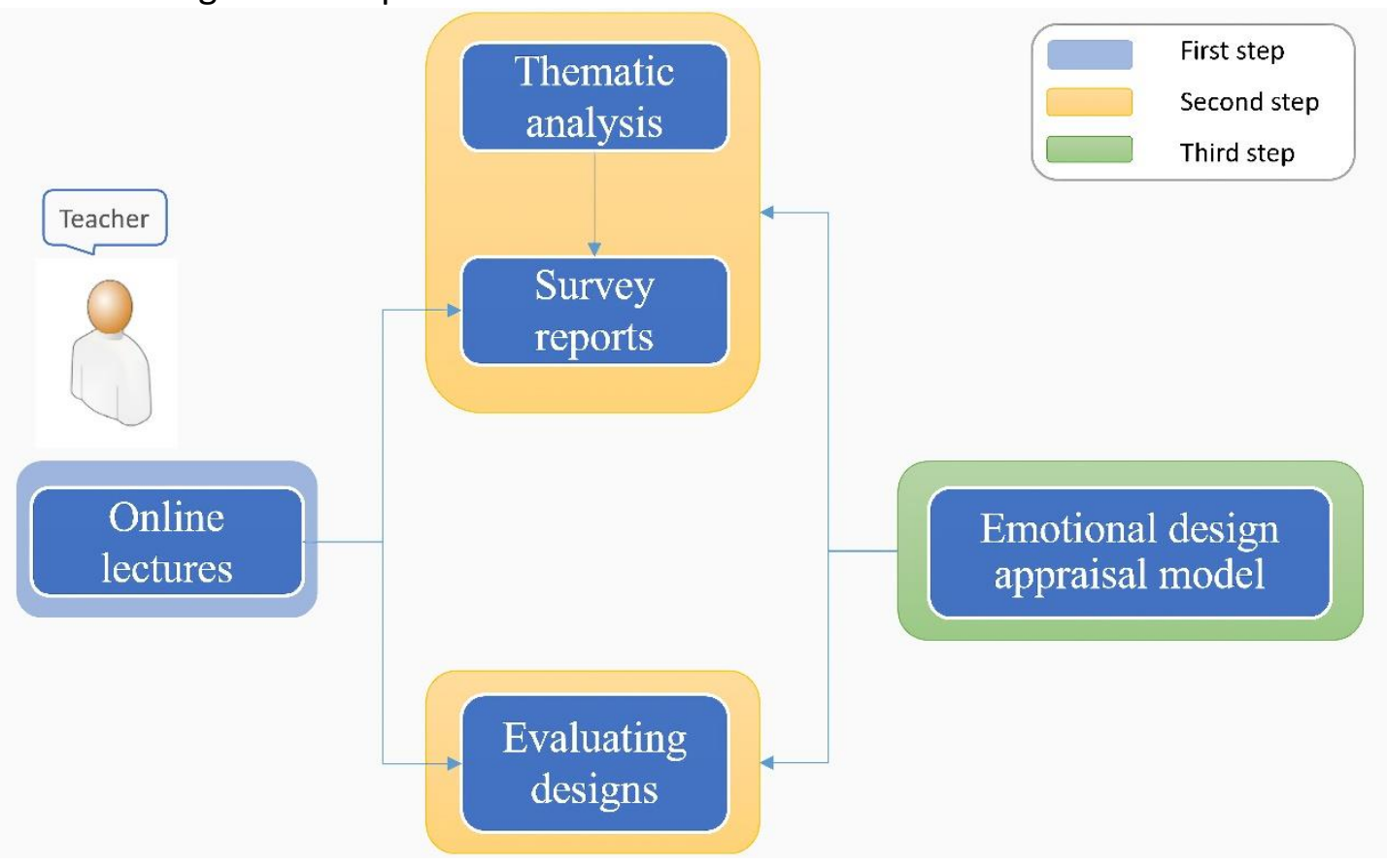

Figure 1: Entire Process of this Case

Source: The Authors Designed the Figure based on the Online Course Plan

\section{Mixed Perspectives}

Mixed perspectives are useful in catering product design to the users' desires from an emotional perspective. Tomico et al. (2012) have defined mixed perspectives in terms of the first, second-, and third-person perspectives. In this case, the first-person perspective is the designer's experience with the product and the users' needs. The designer is normally a current or former user of a similar product. The secondperson perspective is involved with a small group of people in the design process, with the group representing some of the stakeholders. The third-person perspective refers to the designer that she/he has no direct using experiences of the products or services and would start the design work from a broad online survey first.

Influenced by Tomico et al. (2012), in 2016, Smeenk et al. provided an alternative definition of mixed perspectives. Smeenk et al.(2016) conducted a systematic study of mixed perspectives through the case of mourning rituals; this case study included perspective transitions and perspective clusters. A 
perspective transition is a process containing mixed perspectives, and precisely either a combination of different perspectives and/or a switch from one perspective to another. Considering different perspectives in design enabled in-depth investigation of users' requirements.

A mixed perspectives study was also conducted in this hill censer's case study by recording all the transitions and combinations of perspectives in the design process. This study summarises the influence of each perspective and shows the transitions and combinations of perspectives in hill censer design. All these transitions and combinations of mixed perspectives brought new insights to the students in terms of how 'the whole' is not just 'the sum' of individual perspectives.

\section{Thematic Analysis}

Thematic analysis is a process through which patterns or themes are identified within qualitative data (Nowell et al., 2017). As others have pointed out (Astuti, 2020; Boyatzis, 1998; Braun \& Clarke, 2006), thematic analysis is a method for identifying, analysing and reporting themes found in a given phenomenon. Braun and Clarke further developed a six-step approach and demonstrated how to get themes from data in a step-by-step process. This six-step approach is widely considered clear and suitable for developing themes and has generally been influential in the social sciences.

The goal of thematic analysis is to identify themes (Stranges et al., 2014); a theme is considered to be latent content in this method (Vaismoradi \& Snelgrove, 2019). This research applied a qualitative thematic analysis method to extract themes from the data through systematic analysis, which is much more than summarising the data. The survey reports were read carefully, and a five-step method was utilised to generalise nine themes from the survey reports of the students.

\section{Hill Censer Case Study}

The design task required students to write a survey report and design a hill censer for each group in this course. The students learned several methods of surveying and designing in this course, including conducting interviews, framing questionnaires and mixed perspectives. The researcher used the thematic analysis method to analyse the students' survey reports; nine themes were obtained based on Braun's sixstep analysis approach.

\section{Participants}

A total of 42 undergraduate students and ten graduate students (age range was 20-28 years, out of which 38 were females) attended this course, for which they were required to work in groups and design hill censers. The 52 students divided themselves into 18 groups (three or four students per group); each group was required to design a hill censer within the five-week course. The students needed to use multiple methods, especially mixed perspectives, in the design process.

Five professors (three associate professors and two professors) with an industrial design background from the Jingdezhen Ceramic Institute were invited to help with the thematic analysis of the survey reports collected from students. Two online meetings were conducted to discuss the themes that could be generalised from the survey reports. The same five professors and ten censers' users (age range was 30-50, out of which seven were females) evaluated the students' designs. Based on the comments, the researcher graded the design of each group.

\section{Data Collection and Analysis}

The online course lasted five weeks (18 February 2020 to 20 March 2020). The first week consisted of online lectures; the researcher delivered twelve lectures to teach students how to survey and design using multiple methods. The data collection process started in the second week, in which the student groups applied the multiple methods they had learned. They contacted the other product stakeholders to conduct interviews and questionnaires (many of these were held online). The students leveraged those methods to collect the data needed for the design. Each group was required to write a survey report in the third week, which was to 
illustrate the details of the data collection process and the results they obtained.

The qualitative thematic analysis method was employed by the researcher and five professors to extract the themes from the students' survey reports. Five steps of Braun's six-step approach was used in this part of the research. In the first step, the researcher and five professors repeatedly read the survey reports to familiarise themselves with the textual data. In the second step, 86 sub-themes were obtained. During online discussions with the same five professors, 18 sub-themes were deleted since they were similar to other sub-themes, which left only 68 sub-themes. In the third step, the 68 sub-themes were divided into nine themes (categories) in another online meeting with the five professors. Fourth, the researcher reviewed the themes/sub-theme sets to make sure the matching was reasonable. In the final step, the obtained nine themes were defined; these themes and sub-themes were then shared with the students to stimulate them to generate novel ideas.

\section{Findings}

The researcher had online meetings with each group in the third week to gain insights into the impacts of each perspective on the students' design process. The themes and sub-themes were generalised from the students' survey reports, which directly related to the users' opinions; thus, nine themes were obtained. Each theme included several sub-themes, and the interdependence between the sub-themes and the corresponding themes was discussed in depth by the five professors and researcher for confirmation. Finally, the themes were further filtered to reach the core themes.

\section{Impacts of Mixed Perspectives}

Through online meetings with the 18 groups, the teacher observed how the students made the transitions below to change their perspectives during the design processes, which according to Tomico et al. (2012)'s three perspectives are outlined below:

\begin{tabular}{|c|c|}
\hline \multicolumn{2}{|c|}{ The transitions of perspectives during the design processes } \\
\hline $3^{\text {rd }}-2^{\text {nd }}-3^{\text {rd }}-2^{\text {nd }}$ (six groups) & $2^{\text {nd }}-3^{\text {rd }}-2^{\text {nd }}$ (one group) \\
\hline $3^{\text {rd }}-2^{\text {nd }}($ three groups $)$ & $1^{\text {st }}-3^{\text {rd }}-2^{\text {nd }}$ (two group) \\
\hline $3^{\text {rd }}-2^{\text {nd }}-3^{\text {rd }}($ three groups) & $3^{\text {rd }}-1^{\text {st }}-2^{\text {nd }}-3^{\text {rd }}-2^{\text {nd }}$ (one group) \\
\hline $2^{\text {nd }}-3^{\text {rd }}-2^{\text {nd }}-3^{\text {rd }}$ (one group) & $1^{\text {st }}-3^{\text {rd }}-2^{\text {nd }}-1^{\text {st }}$ (one group) \\
\hline
\end{tabular}

Figure 2: The Transitions of Perspectives during the Design Processes

Source: The Author Designed the Figure According to the Results from the Online Meetings.

It is worth mentioning that only four groups used the first-person perspective; those groups included students who had experiences in using censers.

The teacher conducted online meetings with each group to determine the impact of each perspective on the group design.

- Thirteen groups utilised the third-person perspective at the beginning of the design process; it was useful for them in such an early design stage to survey the internet to collect data.
- As for the second-person perspective, the students co-created with stakeholders. Specifically, they investigated the stakeholders and learned how to design hill censers from the users' perspective.

- Four groups used the first-person perspective (designer perspective) to design the hill censers; only a few students had user experience with a censer, and they designed products based on their own experience. 
Most groups considered that the second-person perspective was the most influential. The students reported that they did not know how to design hill censers at the beginning of the course, and most of them had no experience in using censers. In order to understand the users' emotional requirements, the students conducted interviews and questionnaires to cocreate with stakeholders to learn their emotional requirements. Through using these methods, the students could obtain design ideas from the users quickly, and they learned how to design hill censers based on the users' perspectives (second-person perspective).

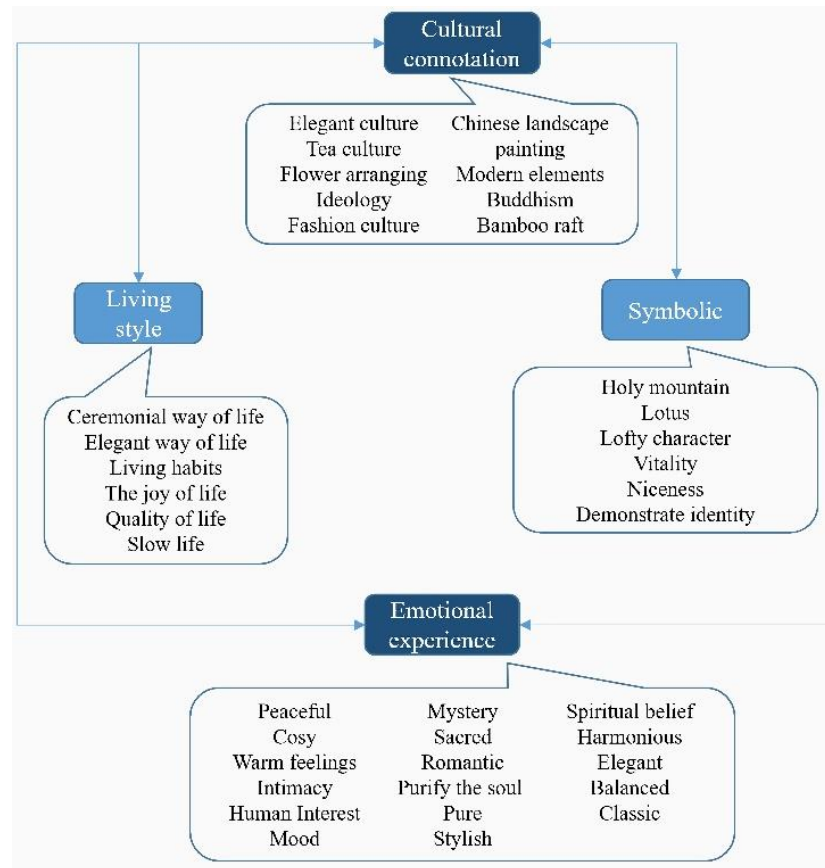

Nine Themes and their Interdependence

The researcher employed the thematic analysis method to study the 18 survey reports. Nine themes were obtained: (1) design method, (2) visual design, (3) function, (4) using occasion, (5) emotional experience, (6) cultural connotation, (7) living style, (8) symbolism and (9) consumer (Error! Reference source not found.). Through online discussions with the five professors, three were selected as the core themes in hill censer design: (1) design strategy, (2) emotional experience, and (3) cultural connotation. The other themes were found to be related to these three core themes during the online discussions with the invited professors.

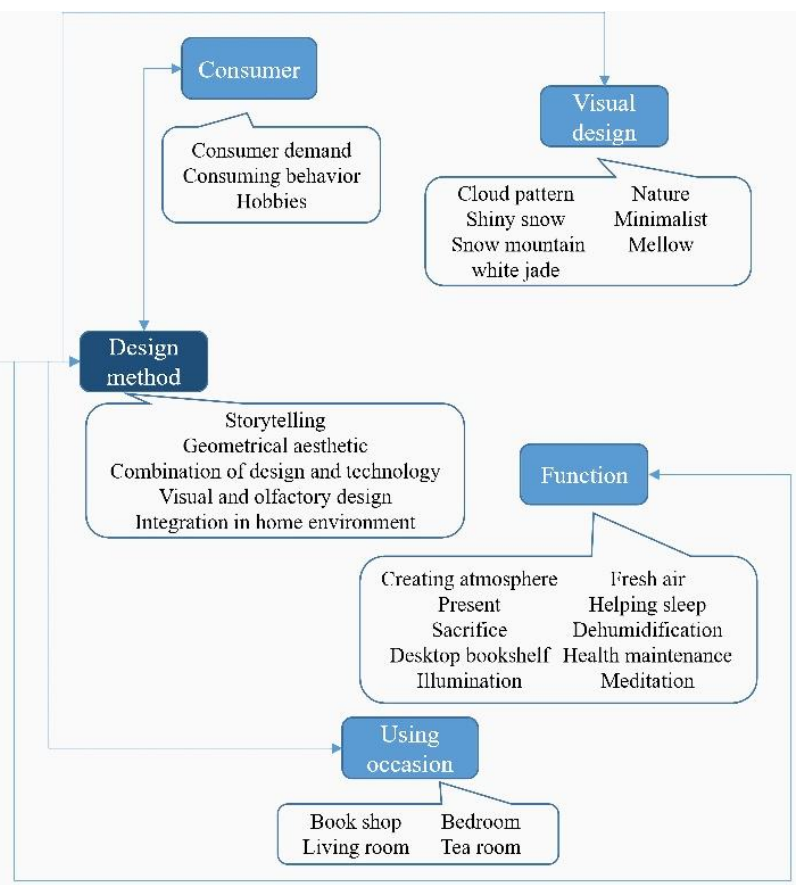

Figure 3: Nine Themes

Source: The Author Designed the Figure According to the Results from the Group Discussions

- First, storytelling is used as a service design tool, which is leveraged to illustrate a scenario where the service is being offered. The students utilised this tool in the hill censer design; it is a new strategy for designing traditional products. Through storytelling, the students understood the censers' using stories; it is useful to design the censer based on users' emotional needs.

- Second, a geometrical aesthetic design method was used, which was influenced by Modernism originating in the Bauhaus design school in the 1920s. In the interviews, some interviewees mentioned they liked censers with a simple form.

- Third, the combination of technology in design was found to be crucial; technology can enhance the positive emotional experience in using products, for instance, by combining interactive technologies with electronic aromatherapy products.

- Fourth, visual and olfactory design was found to be a vital method in censer 
design; the process of using a censer is an incredibly visual and olfactory experience.

- Finally, the integration of the home environment is a new design trend; this means the products should be designed tailored to the home decor trends, such as the censer could be design according to the latest fashion in interior decorating to make the censer more fit to use in the home environment.

The users' 'emotional experience' theme was found to include complicated feelings from the collected data. For instance, sub-themes included 'peaceful', 'cosy', 'warm feelings', 'intimacy' and 'human interest'; these experiences represent users' emotional needs. For example, for users seeking warm feelings, the censer should have been warm-toned to give the users positive feelings. All these sub-themes of emotional experience are related to positive emotions, which means the designers sought out censer designs according to whether they would deliver such positive feelings.

The 'cultural connotation' theme included the following sub-themes: 'elegant culture', 'tea culture', 'flower arranging', 'ideology', 'fashion culture', 'Chinese landscape painting', 'modern elements', 'Buddhism' and 'bamboo raft'. The incense culture included a kind of elegance, which is closely related to tea culture and flower arranging in ancient China (Yu, 2012). Additionally, fashion culture and ideology were also found to influence the design trends of the censers. In the developing history of Chinese censers, the censers of each dynasty were always the embodiment of the fashion and culture of the era; it clearly reflected the ideology of ancient times and conveyed it over time (Shao, 2016). For example, hill censers embodied the auspicious culture and reflected the ruling class's desire to pursue immortality in the Han Dynasty. The other four sub-themes'Chinese landscape painting', 'modern elements', 'Buddhism' and 'bamboo raft' - have typical Chinese culture characteristics and are also related to censer design. From the perspective of users, they realised that the censers are the embodiment of cultural connotation, if the censer has those influences, as mentioned above, could give positive emotional experiences to them.

The themes of 'visual design', 'function', 'using occasion' and 'consumer' are closely related to the 'emotional experience' theme. The visual design theme is more important than the others, incorporating sub-themes like 'cloud patterns', 'shiny white snow', 'snow mountains', 'white jade', 'nature', 'minimalist' and 'mellow'. These sub-themes induce users' emotional interaction, as indicated by the survey reports.

The last two themes, 'living style' and 'symbolism', are both related to the 'emotional experience' and 'cultural connotation' themes, respectively. Users have their own living styles and need products that satisfy their emotional requirements. Symbolism refers to how the form of a product conveys some meaning (Aktar Demirtas et al., 2009). For instance, hill censers symbolically expressed Chinese cultural connotations and signified the immortal desires of the ancient Chinese.

\section{Evaluating the Designs}

The researcher shared the resulting nine themes and sub-themes to the students in the fourth week. Some students were inspired by new design ideas based on these themes. For the remaining two weeks, each group designed a hill censer based on their survey report. The students drew sketches individually and exchanged them with the group. Online group discussions were held to settle on one design for the group and modify the final drawing for submission. The designs submitted were analysed and evaluated against the submitted survey report. Feedback was later given to the students. Five professors evaluated the final submitted designs collaborated with ten users of censers; the evaluation was based on their professional design knowledge and censer-using experience. All groups finished the designs in the fifth week.

The following six designs received the highest scores. These six designs leveraged ideas belonging to the two core themes of 'design 
method' and 'emotional experience'. The evaluators are shown in Figure 3 (in no particular description of each design, as provided by the order).

students, and some comments from the

\section{Designs}

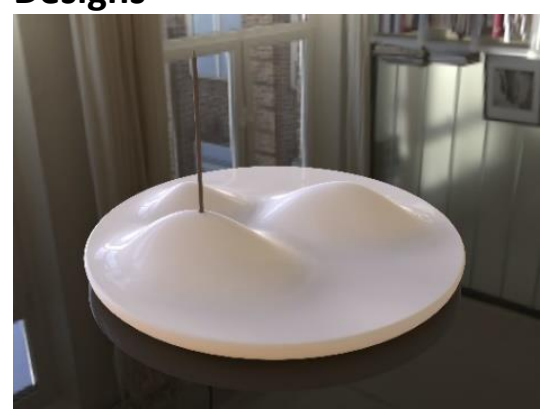

(a)

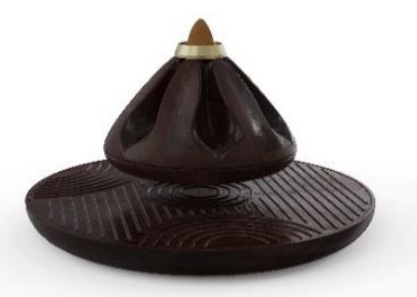

(b)

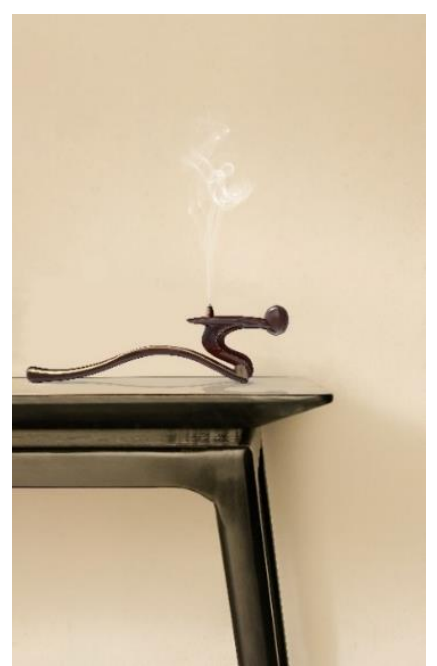

(c)

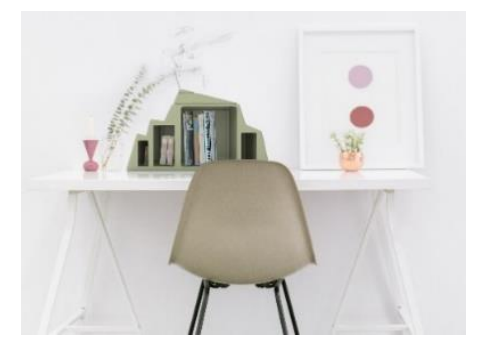

(d)

\section{Design Description}

The ancient Chinese used joss sticks to calculate time as early as a thousand years ago. This incense burner was designed based on this idea. The censer can be used as a time measurement tool when used to burn a joss stick under the sunlight.

The censer is suspended on the wood base, as shown. It utilises the principle of magnetic levitation. The incense mist flows down through the lotus petals' body, which creates the feeling of fairy hills in the sea.

The title of this design is $\mathrm{Mr}$ Fool Wants to Move the Mountain. It is a Chinese folk story in which an older man named Yu Gong, who is almost 90, tries his best to move two high mountains in front of his home. The story tells us that as long as one is persistent, anything can be done.

This censer combines its hill shape with the function of a bookstand. The top is a geometric incense burner that is easily removed for cleaning. The other part is a four-slot bookstand for storing books.

\section{Comments from the Evaluators}

This design uses a simple hill shape, and three hills with different heights make up the censer's body. The pure white glaze colour further complements the beauty of the simple shape of the censer.

This design employs technology. The lotus petals' form expresses a sense of perfume. Magnetic levitation technology is utilised to suspend the censer on the base, which gives the sense that a lotus leaf is holding a flower.

This design is based on a creative idea from an old folk story. Embedded within it is a compound design idea: traditional culture blended in a storytelling approach. The censer takes the form of a person who is carrying a heavy object on his back. The hands of the person extend backwards to keep the 'heavy object' (incense) from falling. The censer is used as a form of a person; Yu Gong is carrying the stones (incense here) in the process of moving the mountains.

The integration of a product with the home environment has been a relatively popular design trend in recent years. This design effectively integrates the product and the home environment since the censer would typically be used in the study. 


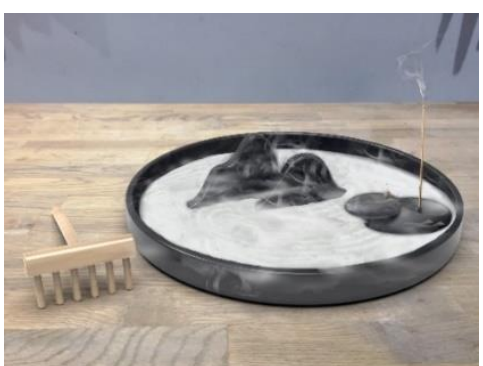

(e)

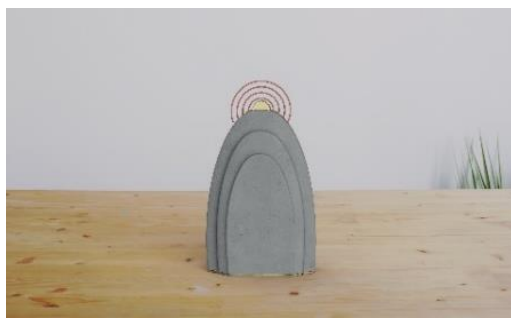

(f)
This design utilises the Japanese rock garden as a creative idea. A rake is designed to pick up the burning incense ashes and make them look like neatly combed sand in a Japanese courtyard, with the 'sand' and black stones mimicking the scene of a Japanese rock garden.

This design utilises a wellknown Chinese short poem about 'Mountain Lu' as a creative idea. The title of the design is Written on the Wall at West Forest Temple by the famous poet Sushi of the Song Dynasty. The abstract form of the censer is used to symbolise Mountain Lu.
The process of using a censer to burn incense is a compound experience based on a visual and olfactory experience. This design utilises a Japanese rock garden to visually express minimalist aesthetics. Additionally, the scented mist will give the user a pleasant experience in terms of smell when using it to burn incense.

The students abstracted the famous Chinese Mountain Lu to create an atmosphere of poetic space. The simple hill shape constitutes the body of the incense burner.

In the artistic design, the discshaped incense and the abstracted shape of the censer form the sunset scene behind the peak of Mountain Lu.

Figure 3: Sample Designs and Comments Source: All the Designs were Assignments for this Online Course

\section{Discussion}

The third step in this case study was to build an emotional design appraisal model, based on the results of the previous steps, to assist designers in coming up with design ideas based on users' emotional requirements, as shown in Figure 4. This model could be used for the design of other kinds of products aside from hill censers. It could also be used to move students to generate novel design ideals quickly and to develop products based on users' emotional requirements. Three stages (pre-design stage, design stage and postdesign stage) are contained in this model (design procedure). In the pre-design stage, starting from the 'design task' as the first step, the students/designers must first understand the design task, and then move on to the next step of survey administration based on the thirdperson perspective, with the purpose being to clarify the research questions. After defining the research questions, co-creation with the stakeholders through the second-person perspective is necessary, and this is counted as the third step. From the angle of the stakeholders, finding answers to the existing questions is the fourth step; these four steps belong to the pre-design stage.

In the design stage, one step of the design work should be done by the students, who should utilise the first-person perspective to design products. The design methods obtained by the thematic analysis method could be used to help the students easily come up with novel design ideas. However, the process should not be limited to these methods, since any method that can help to generalise the design ideals would be feasible for use in the design stage.

In the post-design stage, students need to get feedback from the stakeholders. If the results are positive, then they can move to the next step, but if the results are negative, they should go back to step two or five to redesign the products. 


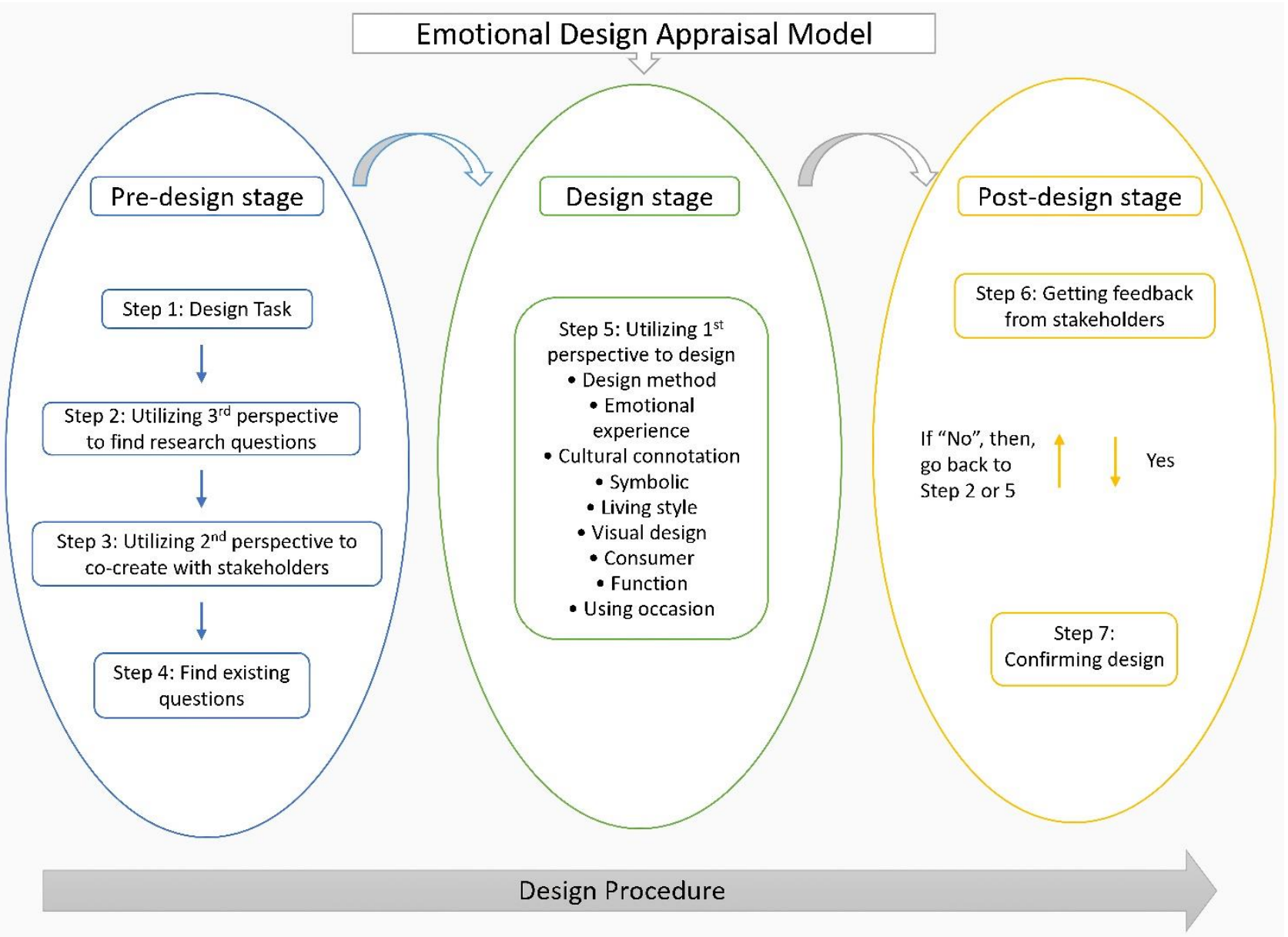

Figure 4: Emotional Design Appraisal Model Source: The Author Designed the Figure According to the Results from this Case Study

\section{Conclusion}

Two main findings were obtained in this research. First, all the groups' designs involved the stakeholders, the second-person perspective has the most apparent influence on the students' designs than other perspectives. The students with stakeholders co-created based on users' emotional requirements after learning about censer and incense use. As it turned out by this case study, adopting the second-person perspective (stakeholders' perspective) can allow the students to quickly generate design ideas.

Second, the 'design method' and 'emotional experience' themes obtained by the thematic analysis were found to be useful in the product design of the hill censer. Designs with these characteristics received high scores from the users and experts (professors). This means that these two themes are more important than others in product design. The 'design method' theme in this study can guide students in designing from innovation design perspective. Specifically, this theme includes five sub-themes that can help designers obtain novel design ideas. The 'emotional experience' theme can guide designers to understand the emotional needs of users in detail. This theme incorporates 17 sub-themes, and designers can learn more about the users' emotional requirements through its implementation.

The limitation of this research is that the emotional design appraisal mode still needs to be evaluated with other kinds of products to test its feasibility in future work.

\section{References}

Aktar Demirtas, E., Anagun, A. S., \& Koksal, G. (2009). Determination of optimal product styles by ordinal logistic regression versus conjoint analysis for kitchen faucets. International Journal of Industrial Ergonomics, 39(5), 866875. 
https://doi.org/10.1016/j.ergon.2009.06.007

Astuti, T. (2020). The dynamic of performing social relations amongst managers and supervisors : A Thematic Analysis. TQR: Where the World Comes to Learn Qualitative Research. 25(3), 700-720.

https://nsuworks.nova.edu/tqr/vol25/iss3/10/

Braun, V., \& Clarke, V. (2006). Using thematic analysis in psychology. Qualitative Research in Psychology, 3(2), 77-101.

https://doi.org/10.1191/1478088706qp063oa

Boyatzis, R. E. (1998). Transforming qualitative information: Thematic analysis and code development. Thousand Oaks, CA: Sage.

Lin X. J., (2008). Boshanlu Verificine. Sichuan Cultural Relics. 3, 65-67.

Nowell, L. S., Norris, J. M., White, D. E., \& Moules, N. J. (2017). Thematic Analysis: Striving to Meet the Trustworthiness Criteria.

International Journal of Qualitative Methods, 16(1), 1-13.

https://doi.org/10.1177/1609406917733847

Sáenz, D. C., Domínguez, C. E. D., LlorachMassana, P., García, A. A., \& Arellano, J. L. H. (2019). A series of recommendations for industrial design conceptualizing based on emotional design. Managing Innovation in Highly Restrictive Environments, 167-185

Shao, X. L., (2016). A renewed study on Boshan incense burners: origins and other issues. Journal of National Museum of China, 5, 52-61. Shroyer, K., Lovins, T., Turns, J., Cardella, M. E., \& Atman, C. J. (2018). Timescales and ideaspace: An examination of idea generation in design practice. Design Studies, 57, 9-36. https://doi.org/10.1016/j.destud.2018.03.004

Smeenk, W, Tomico, O., Turnhout, van, Smeenk, W., \& Tomico, O. (2016). A systematic analysis of mixed perspectives in empathic design: not one perspective encompasses all. International Journal of Design, 10(2), 31-48.

Stranges, M. K. W., UI Haq, S., \& Dunn, D. G. (2014). Black-out test versus UV camera for corona inspection of HV motor stator endwindings. IEEE Transactions on Industry
Applications, 50(5), 3135-3140.

https://doi.org/10.1109/TIA.2014.2306979

Tomico, O., Winthagen, V. O., \& Van Heist, M.

M. G. (2012). Designing for, with or within: 1st,

2nd and 3rd person points of view on designing for systems. NordiCHI 2012: Making Sense Through Design - Proceedings of the 7th Nordic Conference on Human-Computer Interaction, 180-188. https://doi.org/10.1145/2399016.2399045

Vaismoradi, M., \& Snelgrove, S. (2019). Theme in qualitative content analysis and thematic analysis. Forum Qualitative Sozialforschung, 20(3). https://doi.org/10.17169/fqs-20.3.3376

Van Gorp, T., \& Adams, E. (2012). Design for Emotion. Elsevier

$\mathrm{Yu}, \mathrm{Q}$. H., (2012). The research about the art of creation of censer from the Warring States Period to the Song Dynasty. Beijing Arts and Crafts Press.

\section{Conflict of Interest Statement}

The authors report no potential conflict of interest

\section{Acknowledgements}

This research was supported by the Art General Project of 2018 National Social Science Fund (18BG118, Research on the History of Chinese Traditional Tool Design Concepts). We also thank the anonymous reviewers for the valuable constructive suggestions.

\section{Authors' Contributions}

The contributions of the respective authors are as follows:

YU Qinghua: conducting the online course and group discussions; generalising the data; developing the first draft and methodology; contribution in validation of data.

Yukari Nagai: Guidance and supervision; developing the final draft alongside editing and reviewing.

The datasets generated during the current study are available from the corresponding author on reasonable request. 